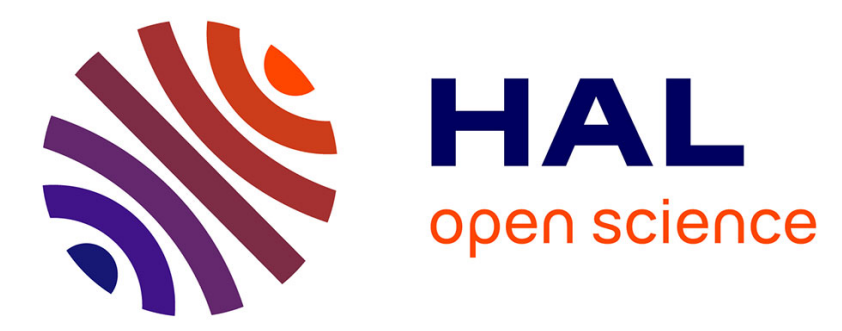

\title{
High-efficiency subwavelength diffractive element patterned in a high-refractive-index material for $633 \mathrm{~nm}$
}

Simion Astilean, Philippe Lalanne, Pierre Chavel, Edmond Cambril, Huguette

\section{Launois}

\section{- To cite this version:}

Simion Astilean, Philippe Lalanne, Pierre Chavel, Edmond Cambril, Huguette Launois. Highefficiency subwavelength diffractive element patterned in a high-refractive-index material for $633 \mathrm{~nm}$. Optics Letters, 1998, 23 (7), pp.552-554. 10.1364/OL.23.000552 . hal-00877412

HAL Id: hal-00877412

https://hal-iogs.archives-ouvertes.fr/hal-00877412

Submitted on 28 Oct 2013

HAL is a multi-disciplinary open access archive for the deposit and dissemination of scientific research documents, whether they are published or not. The documents may come from teaching and research institutions in France or abroad, or from public or private research centers.
L'archive ouverte pluridisciplinaire HAL, est destinée au dépôt et à la diffusion de documents scientifiques de niveau recherche, publiés ou non, émanant des établissements d'enseignement et de recherche français ou étrangers, des laboratoires publics ou privés. 


\title{
High-efficiency subwavelength diffractive element patterned in a high-refractive-index material for $633 \mathrm{~nm}$
}

\author{
Simion Astilean, ${ }^{*}$ Philippe Lalanne, and Pierre Chavel \\ Institut d'Optique Théorique et Appliquée, Centre National de la Recherche Scientifique, B.P. 147, F-91403 Orsay Cedex, France \\ Edmond Cambril and Huguette Launois \\ Laboratoire de Microstructures et de Microélectronique, L2M Centre National de la Recherche Scientifique, \\ 196 avenue Henri Ravera, B.P. 107, F-92225 Bagneux, France
}

Received November 25, 1997

\begin{abstract}
We propose the use of high-index materials for the fabrication of subwavelength diffractive components operating in the visible domain. This approach yields a reduction of fabrication constraints and an improvement of theoretical performance. A blazed grating with subwavelength binary features and with a period of 5.75 wavelengths is designed and fabricated in a $\mathrm{TiO}_{2}$ layer coated upon a glass substrate. The first-order diffraction efficiency measured with a $\mathrm{He}-\mathrm{Ne}$ laser beam is $83 \%$, which is slightly larger than that achieved theoretically by the best standard (continuous profile) blazed grating fabricated in glass with the same period. (C) 1998 Optical Society of America

OCIS codes: $\quad 050.2770,050.1970$.
\end{abstract}

With the progress of nanofabrication technologies, it was recently predicted ${ }^{1,2}$ that surface-relief diffractive elements with subwavelength binary features may exhibit high diffraction efficiency $(\approx 90 \%)$ for visible-light operation. These high-frequency gratings exploit the analogy between subwavelength structures and homogeneous media to synthesize artificial dielectric materials in which the local effective index is related to the fraction of matter etched. Therefore the design and fabrication of these components have received much attention. ${ }^{3-9}$ The first subwavelength phase-plate element operating at a wavelength of $633 \mathrm{~nm}$ was etched in a fused-quartz substrate. ${ }^{7}$ By varying the fill factors of square pillars engraved upon a 400-nm grid, Chen and Craighead synthesized six effective index values to produce a first-order blazed grating. The fabricated grating exhibited a 55\% diffraction efficiency. In a subsequent paper by the same authors, a diffractive lens operating at $633 \mathrm{~nm}$ and etched in fused quartz was reported. ${ }^{8}$ To relax the fabrication constraints, a 700-nm grid spacing was chosen, and a $3 \pi / 2$ phase element (instead of $2 \pi$ ) was fabricated. The diffraction efficiency measured in the focal spot of the lens was $53 \%$. Later, Miller et al. reported ${ }^{9}$ on a $74 \%$ diffraction-efficiency 1-to-3 fanout element. This element, which was fabricated into photoresist, was operating at $633 \mathrm{~nm}$ for TE polarization. Although it exhibits the highest reported diffraction efficiency for visible-light operation, it is noteworthy that its operation requires only a $\pi$ phase-shift modulation. For the sake of completeness, let us mention the fabrication of a blazed transmission grating operating for TE polarization at $975 \mathrm{~nm}$. This grating exhibited a $66 \%$ diffraction efficiency in the first order. ${ }^{5}$ Clearly, this overview shows that the performance experimentally achieved by subwavelength diffractive elements is lower than that theoretically predicted or obtained by standard diffraction elements with continuous blazed profiles. The major difficulty comes from the extremely high aspect ratios of the groove depth to the minimum transverse feature size required for a $2 \pi$ phase shift modulation.

To realize fully the potential of subwavelength diffractive elements, it is important to reduce the aspect-ratio requirements. In general terms our idea is that one can circumvent these requirements by engraving high-refractive-index materials. If a subwavelength structure is etched into a material of refractive index $n$ and depth $h$, the phase shift $\phi$ experienced by a light beam with a wavelength $\lambda$ in vacuum is $\phi=(2 \pi / \lambda)\left(n_{\text {eff }}-1\right) h$. By controlling the fraction of removed material, one can vary $n_{\text {eff }}$ between $n$ and the index of the incident medium (supposedly 1), and a $2 \pi$ phase-shift modulation is achieved for a depth equal to $\lambda /(n-1)$. Therefore the depth and consequently the aspect ratio are reduced when high-index materials are used. In this Letter we report on the design, fabrication, and test of a high-efficiency, $2 \pi$ phase-shift subwavelength diffractive element etched in a high-refractive-index layer $\left(\mathrm{TiO}_{2}\right)$ for $633 \mathrm{~nm}$.

However, a problem arises, namely, that Fresnel losses that are due to the high-index material coated upon the low-index substrate may reduce the transmitted signal. To address this question we consider the following index-modulated grating; along the period, the refractive index varies linearly from 1 to a maximum value $n$. In this case the grating is a periodic structure with a real graded index, and no subwavelength features that mimic effective media are considered. A plane wave illuminates the grating from air at normal incidence. The substrate is glass $\left(n_{g}=1.52\right)$. The grating thickness and period are $\lambda /(n-1)$ and $5.75 \lambda$, respectively. The diffraction problem is analyzed for TM polarization with rigorous coupled-wave analysis. ${ }^{10}$ In Fig. 1 the solid curve represents the first-order diffraction efficiency as a function of $n$. Open circles represent the percentage of the total transmitted light diffracted into the first order, an important figure of merit related to the amount of noise created by the spurious diffracted orders. Clearly, the 


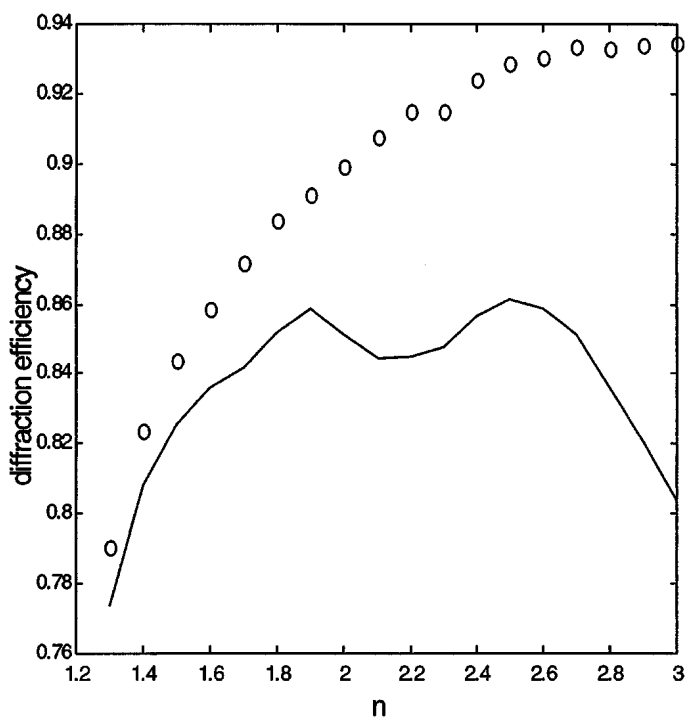

Fig. 1. Diffraction efficiency (solid curve) for TM polarization as a function of the maximum index $n$ for a linearly index-modulated grating. The grating period is $5.75 \lambda$. The circles represent the percentage of the total transmitted light diffracted into the first order.

use of high-index material has a beneficial effect on this percentage and reduces noise. The dependence on $n$ of the diffraction efficiency is governed basically by the grating antireflection properties. These properties explain the corrugated shape observed for $n$ values of 1.8-2.6. For $n$ equal to 1.9 and 2.5, which correspond to two local maxima of the diffraction efficiency, the grating reflectivity is minimal. For $n$ larger than 2.6, the diffraction efficiency drops because of increasing Fresnel losses.

In this study a blazed grating with subwavelength features operating under TM polarization at $633 \mathrm{~nm}$ is considered. As a high-refractive-index material, we chose $\mathrm{TiO}_{2}$ because it is a common coating material for thin-film applications. Its refractive index is $n=$ 2.30. Thus, in comparison with glass material $(n \approx$ 1.5), for $\mathrm{TiO}_{2}$ a reduction of a factor of 2.6 in the aspect ratio is expected. We chose a blazed grating for the demonstration because it requires full-range $(0-2 \pi)$ phase-shift modulation while offering simple design procedure and device characterization. The grating period is $3.64 \mu \mathrm{m}$ (nearly $10^{\circ}$ deflection in air). We used a depth value of $540 \mathrm{~nm}$, slightly larger than required by scalar theory $[\lambda /(n-1)=487 \mathrm{~nm}]$ to relax the fabrication constraints of narrow lines related to small effective indices. A subwavelength period of $405 \mathrm{~nm}$, just shorter than the cutoff value $(\lambda / n=416 \mathrm{~nm})$ at normal incidence, was chosen to ensure that all diffracted orders, except the zeroth transmitted and reflected orders, are evanescent. For this value the grating period is divided exactly into nine subwavelength periods. The design proceeded as follows. First, we computed the fill factors of the nine grooves, using effective-medium theory. ${ }^{11}$ The resulting design is shown as profile (a) in Fig. 2. According to rigorous coupled-wave computation, the first-order diffraction efficiency of this grating is $87 \%$. Second, because of severe fabrication constraints on the minimum groove, the narrowest groove [at the left in profile (a) of Fig. 2] was removed. Finally, we optimized the 16 remaining transition-point locations, using a gradient-descent algorithm to maximize the first-order diffraction efficiency. As can be seen from profile (b) of Fig. 2, which shows the final grating structure, only a few modifications were obtained. We ended up with a theoretical diffraction efficiency of 91\%. Approximately $3 \%$ of the light was diffracted into spurious transmitted orders; the remaining $6 \%$ was backreflected. Normal incidence from air was assumed for the design. Similar results were obtained for normal incidence from the glass substrate. The minimum linewidth was $101 \mathrm{~nm}$, the minimum groove width was $118 \mathrm{~nm}$, and the maximum aspect ratio was 5.3 , a value significantly smaller than those used in the research reported in Refs. 7-9.

An electron-beam evaporation technique with a plasma gun was used for the $\mathrm{TiO}_{2}$ coating. The plasma was composed of a $42 / 58 \mathrm{Ar} / \mathrm{O}_{2}$ gas combination. The full evaporation process was optimized to yield stable and dense layers. The deposition rate was approximately $0.1 \mathrm{~nm} / \mathrm{s}$. The $\mathrm{TiO}_{2}$ layer deposited upon a glass substrate was patterned by a combination of electron-beam lithography and reactive ion etching. First, a 150-nm-thick poly(methyl methacrylate) layer was spin coated on top of the $\mathrm{TiO}_{2}$ layer. Pattern generation was performed with a JEOL JBX5D2U vector scan high-resolution pattern generator equipped with an LaB6 filament. Exposure was performed with a $50-\mathrm{keV}$ electron beam with a 25 -nm-diameter probe beam. After the pattern was developed, an intermediate 40-nm-thick $\mathrm{Ni}$ layer was electron-beam evaporated onto the surface and lifted off by dissolution of the poly(methyl methacrylate). The lift-off technique was used to improve the selectivity and the fidelity of the pattern transfer during the $\mathrm{TiO}_{2}$ etching process, which consisted of reactive ion etching in a Nextralne 110 system equipped with a $\mathrm{Si}$ cathode. The etching process used a $\mathrm{SF}_{6}(1 / 2) / \mathrm{CH}_{4}(1 / 2)$ gas mixture at a pressure of $8 \mathrm{mTorr}(1.07 \mathrm{~Pa})$ with the

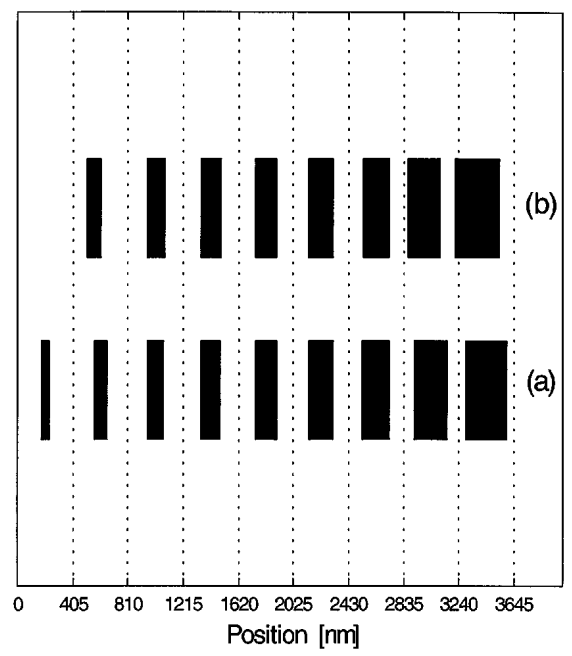

Fig. 2. Grating profiles for different steps of the design. Only one grating period is shown: (a) from effective medium theory, (b) after refinement of the transition-point locations. 


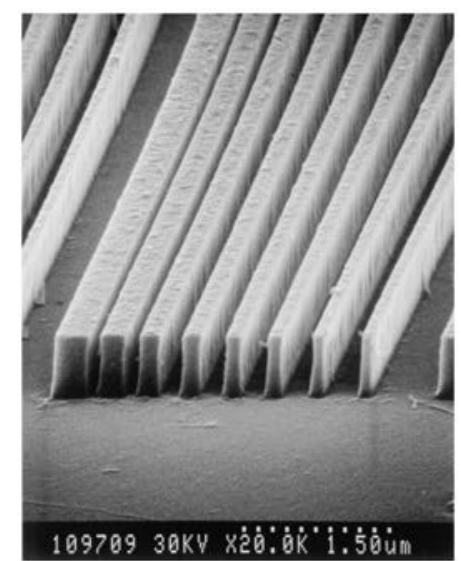

Fig. 3. Scanning-electron micrograph of a single period of the subwavelength blazed grating.

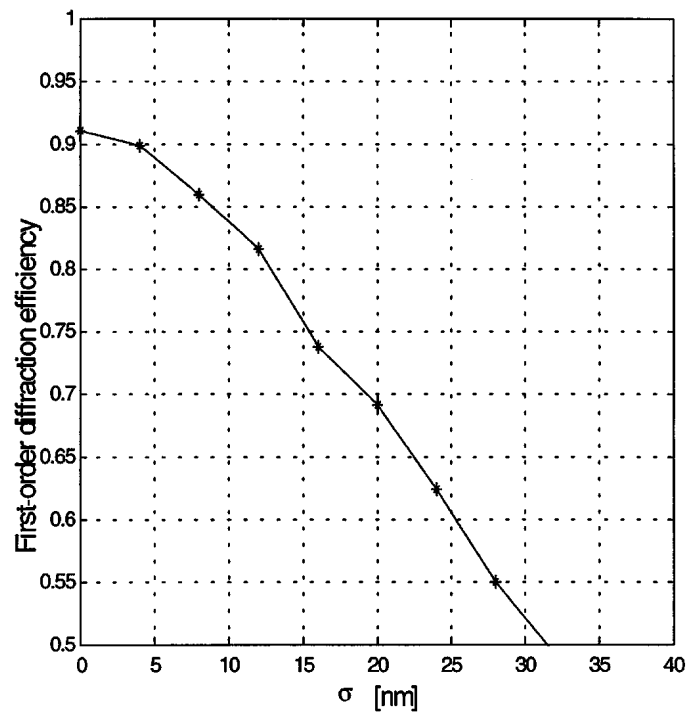

Fig. 4. First-order diffraction efficiency as a function of the standard error deviation of linewidths. Each point is averaged over 100 independent instances of fabrication errors.

same flow for each gas and a rf power of $30 \mathrm{~W}$, which produced a self-induced bias voltage of $-180 \mathrm{~V}$. This process, optimized for steeper sidewalls, resulted in an etching rate of $40 \mathrm{~nm} / \mathrm{min}$. The grating pattern was written over a $160 \mu \mathrm{m} \times 160 \mu \mathrm{m}$ area, which is four times larger than the writing field of the $e$-beam generator operating at its highest resolution. A scanning-electron photograph of the grating is shown in Fig. 3.

The grating testing was carried out with a $\mathrm{He}-\mathrm{Ne}$ laser under TM polarization and at normal incidence from air. We determined the diffraction efficiencies by measuring the powers of the diffracted beams and dividing them by the power of the incident beam. We corrected the measurements by including the Fresnel losses at the back side of the glass substrate. We observed that $92 \%$ of the incident light is transmitted, $5 \%$ is reflected, and the remaining $3 \%$ is scattered. The diffraction efficiency of the first order was $83 \%$, a value $8 \%$ lower than the theoretical prediction. We also observed that $90 \%$ of the total transmitted light is diffracted into the first order, compared with $97 \%$ predicted by electromagnetic theory. The sensitivity of linewidth fabrications errors was also investigated, under the assumption that the center locations of the grooves are positioned accurately. We assumed that the linewidths are independent Gaussian-distributed variables, with mean values the nominal linewidths and with standard deviation $\sigma$. Clearly, this model is unsatisfactory, but we believe that it provides a first insight into the sensitivity of the subwavelength grating to fabrication errors. Figure 4 shows the first-order diffraction efficiency as a function of $\sigma$. Note that an efficiency reduction from $91 \%$ to $83 \%$ is obtained for $\sigma=12 \mathrm{~nm}$, a value approximately equal to one tenth of the minimum linewidth.

To our knowledge the blazed grating with subwavelength features reported here exhibits the highest efficiency $(83 \%)$ yet reported for visible-light operation. Moreover, it is noteworthy that the maximum diffraction efficiency achieved by a standard blazed grating (continuous triangular profile) with a $5.75 \lambda$ period is $82 \%$, a theoretical value slightly lower than that obtained experimentally in this research. This efficiency computed with electromagnetic theory holds for a grating etched into glass $\left(n_{g}=1.52\right)$, an optimized grating depth equal to $1.06 \lambda /\left(n_{g}-1\right)$, TM polarization, and normal incidence from air.

This research was supported by the European Community under the RODCI Mel-Ari program. The authors are grateful to Jean Landreau and Alain Carenco of the Centre National des Etudes de Télécommunications-Bagneux for coating the $\mathrm{TiO}_{2}$ layer. S. Astilean is pleased to acknowledge financial support from NATO.

*Present address, Department of Optics and Spectroscopy, Faculty of Physics, Babes-Bolyai University, 3400 Cluj-Napoca, Romania.

\section{References}

1. W. Stork, N. Streibl, H. Haidner, and P. Kipfer, Opt. Lett. 16, 1921 (1991).

2. M. W. Farn, Appl. Opt. 31, 4453 (1992).

3. H. Haider, J. T. Sheridan, and N. Streibl, Appl. Opt. 32, 4276 (1993).

4. Z. Zhou and T. J. Drabik, J. Opt. Soc. Am. A 12, 1104 (1995).

5. M. E. Warren, R. E. Smith, G. A. Vawter, and J. R. Wendt, Opt. Lett. 20, 1441 (1995).

6. P. Kipfer, M. Collischon, H. Haidner, and J. Schwider, Opt. Eng. 35, 726 (1996).

7. F. T. Chen and H. C. Craighead, Opt. Lett. 20, 121 (1995).

8. F. T. Chen and H. C. Craighead, Opt. Lett. 21, 177 (1996).

9. J. M. Miller, N. de Beaucoudrey, P. Chavel, E. Cambril, and H. Launois, Opt. Lett. 21, 1399 (1996).

10. Ph. Lalanne and G. M. Morris, J. Opt. Soc. Am. A 13, 779 (1996).

11. S. M. Rytov, Sov. Phys. JETP 2, 466 (1956). 